\title{
Optimization Design Method of Optical Remote Sensor Based on Imaging Chain Simulation
}

\author{
Xing Kun ${ }^{\mathrm{a}}$, Cao Shi-xiang, Yue Chun-yu, Zhou Nan and He hong-yan \\ Beijing Institute of Space Mechanics \& Electricity, Beijing 100094, China
}

\begin{abstract}
Space optical remote sensor of high spatial resolution need achieve both light and small design and high imaging quality. An optimization method of optical remote sensor based on imaging chain simulation is presented. All factors in imaging chain are analyzed according to MTF models and noise models. Additionally, image restoration is considered as one part of imaging chain. The simulation model are built to optimize remote sensor overall performance by the evaluation of remote sensor and simulation image. This method is used to enhance image quality and reduce the development difficulties of optical remote sensor. The test validates the feasibility and predominance of the optimization design method.
\end{abstract}

\section{Introduction}

Many countries in the development of remote sensing imaging system, attach great importance to the simulation analysis. With the development of military, civil and commercial remote sensing satellites, the United States has put forward a series of CONOP (Concept of Operation), SSAD (Space System Analyses and Design) and performance simulation and application simulation systems, which promotes the design, manufacture and application of remote sensing satellite system[1].1995, LaRC (Landley Research Center) funded by NASA announced the PATCOD integrated design software platform [2]. It combines Pro/Engineer structural design, Pro/Manufacturing, PATRAN, NASTRAN, SINDA-85, P/Thermal, CODE V and other common software for spacecraft simulation design and analysis process. The system-level simulation software as developed by Alcatel, France, has been used to simulate the optical system of the earth observation, and has been widely applied in MERIS (ENVISAT), SEVIRI（MSG）, HRG（SPOT） and HR（Pleiades）. In addition, Kodak has developed Physique software. In the image quality assessment and estimation, the U.S. government's IRARS and its collaborators jointly developed the NIIRS standard $[3,4]$. The GIQE equation provides a forecast for the NIIRS grade [5,6], performance prediction of the remote sensing system can be given in remote sensing system designing and parameters, the quality assessment of remote sensing image can be given before system development [7], so as to optimize the design of remote sensor. In the process of Pleiades system demonstration, France has made a comprehensive balance of several important parameters (MTF, SNR, compression ratio, deconvolution, de noise, etc.)

\footnotetext{
${ }^{a}$ Corresponding author: xingkunfeixiang@aliyun.com

Fund Project: China 973 Program (61321001), High Resolution Earth Observation Special Fund

(GFZX40136-03-02), National natural science foundation(41401411)
} 
which affect the final image quality, through the system optimization and the ground comprehensive processing, the image quality can be guaranteed [8]. It can be seen from the optimization methods, MTF and SNR are important factors affecting image quality. An optimization method of optical remote sensor based on imaging chain simulation is presented.

\section{Optical remote sensing simulation model}

Imaging simulation are realized through researching the influence of $3 \mathrm{~d}$ scenes, imaging conditions (The atmosphere and background light, etc.), remote sensing (The optical system, detector, circuit), satellite platform, data transmission, image restoration, etc. Path of Remote sensor imaging simulation technology is shown in Figure 1.

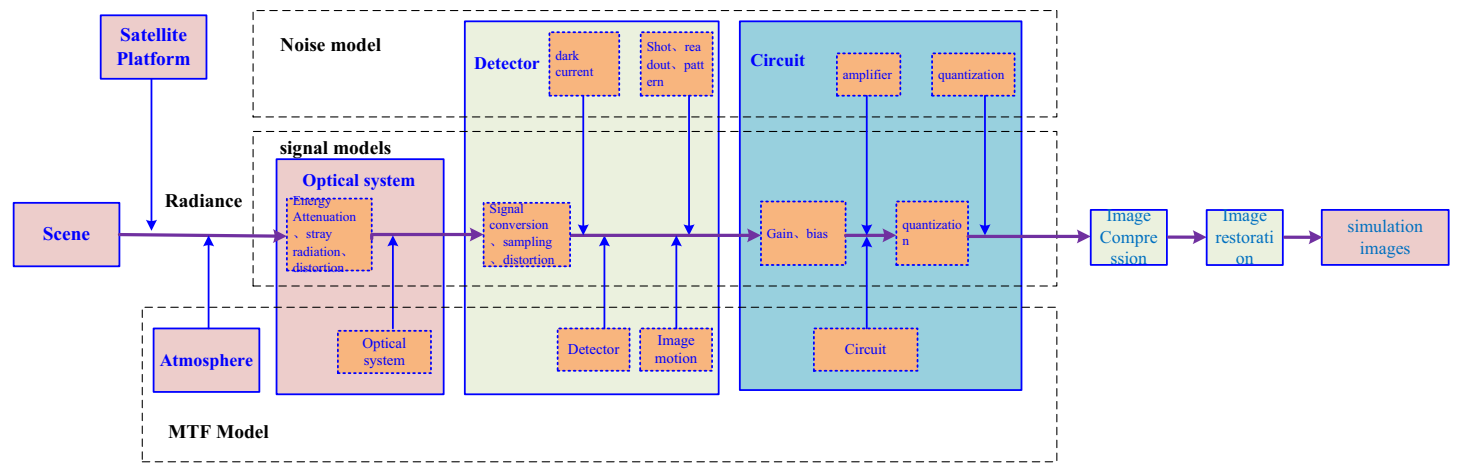

Figure 1. Optical remote sensing imaging simulation technology roadmap

\subsection{Scene}

Scene is consists of $3 \mathrm{~d}$ scene and the target source geometry characteristics, physical properties and thermal characteristics and so on. $3 \mathrm{~d}$ scenes target source geometry is based on the geometric module development.

\subsection{Atmosphere}

The MTF model established in this paper, considers atmospheric degradation characteristics of imaging system. The main consideration is turbulence MTF and aerosol MTF. For space optical remote sensing applications, the turbulent MTF in the far field and short exposure condition can refer to literature $[9,10]$. Aerosol impact on image comes mainly from low altitude atmosphere part. MTF of degeneration of imaging system cause by turbulence and aerosols is:

$$
M T F_{\text {atm }}=M T F_{\text {turb-se }} \times M T F_{\text {aerosol }}
$$

In the last formula, $M T F_{\text {turb-se }}$ is atmospheric turbulence MTF, $M T F_{\text {aerosol }}$ is aerosol MTF.

\subsection{Remote Sensor}

Remote sensor is mainly composed of optical system, detector and circuit. The optical system MTF model can be represented by the type:

$$
M T F_{\text {opt }}=M T F_{\text {diff }} \times M T F_{\text {aberration }} \times M T F_{\text {defocus }} \times M T F_{\text {other }}
$$

$M T F_{\text {diff }}$ is the optical system MTF, divided into the central obscuration and no central obscuration of the circular aperture diffraction. $M T F_{\text {aberration }}$ is the optical system aberration MTF. MTF defocus is the focal shift MTF. In addition to the above factors, there are parts processing error, installation error, etc. 
Line-Array CCD two directions of MTF are as follows:

$$
\begin{gathered}
\operatorname{MTF}_{\text {DETECTOR_CCD }_{-}\left(f_{x}\right)=\operatorname{MTF}_{S L} \times M T F_{\text {other }}} \\
\operatorname{MTF}_{\text {DETECTOR_CCD }_{-}\left(f_{y}\right)=} \operatorname{MTF}_{S L}\left(f_{y}\right) \times \operatorname{MTF}_{\text {DIFFUSION }}\left(f_{y}\right) \times \operatorname{MTF}_{\text {TRANSFER }}\left(f_{y}\right) \times \operatorname{MTF}_{\text {other }}
\end{gathered}
$$

$\mathrm{X}$ direction is the direction of satellite flight. $\mathrm{Y}$ direction is the direction of detector array. $M T F_{\text {DETECTOR_CCD }_{-}}$is detector MTF. $M T F_{S L}$ is detector geometry MTF. MTF $F_{\text {DIFFUSION }}$ is photoelectron diffusion MTF. MTF $F_{\text {TRANSFER }}$ is charge transfer MTF. MTF degradation induced by other factors satisfies Gauss's function.

There are four main types of detector noise: shot noise, dark current noise readout noise and mode noise. The noise size is represented by the RMS noise equivalent electron number. The detector noise is calculated as:

$$
N_{\text {detector_rms }}=\sqrt{\left(N_{\text {shot_rms }}\right)^{2}+\left(N_{\text {dark_rms }}\right)^{2}+\left(N_{\text {readout_rms }}\right)^{2}+\left(N_{\text {mode_rms }}\right)^{2}}
$$

The circuit model is mainly represented by the electronic filter MTF. The noise model of the circuit needs to consider the amplifier noise and quantization noise.

\subsection{Satellite Platform}

Single integral time is $T$, normal push scan speed is $v$, pixel center spacing is $p=v T$. The initial integration time at level $n$ of line $L$ is $(L+n-2) T$. Suppose the abnormal image motion in the TDI direction is $X(t)$, then the image motion at level $n$ integration time of line $L$ in the TDI direction is $\xi x(t)=v[t-(L-2+n) T]+X(t)$. Suppose the abnormal image motion in vertical TDI direction is $Y(t)$, then $\xi y(t)=Y(t)$. The image motion of different lines is inconsistent. The resulting image quality decline is spatially varying, which cannot be directly obtained by convolution with a PSF and the image. The image needs to be calculated in the sub-region by convolution. The line spread function in the TDI direction is ( $\delta(x)$ is Dirac function)

$$
\begin{aligned}
\mathrm{LSF}_{x} & =\frac{1}{N T} \sum_{n=1}^{N} \int_{(L+n-2) T}^{(L+n-1) T} \delta(x-v(t-(L+n-2) T)-X(t)) \mathrm{d} t \\
& =\frac{1}{N T} \sum_{k=L-1}^{N+L-2} \int_{k T}^{(k+1) T} \delta(x-v(t-k T)-X(t)) \mathrm{d} t
\end{aligned}
$$

The line spread function in the vertical TDI direction is

$$
\begin{aligned}
\operatorname{LSF}_{y} & =\frac{1}{N T} \sum_{n=1}^{N} \int_{(L+n-2) T}^{(L+n-1) T} \delta(y-Y(t)) \mathrm{d} t \\
& =\frac{1}{N T} \int_{(L-1) T}^{(L+N-1) T} \delta(y-Y(t)) \mathrm{d} t
\end{aligned}
$$

The MTF in two directions is obtained by Fourier transform of the diffusion function.

$$
\begin{gathered}
\mathrm{MTF}_{x}=\frac{1}{N T}\left|\sum_{k=L-1}^{N+L-2} \int_{k T}^{(k+1) T} \exp \{-\mathrm{j} 2 \pi f[v(t-k T)+X(t)]\} \mathrm{d} t\right| \\
\mathrm{MTF}_{y}=\frac{1}{N T}\left|\int_{(L-1) T}^{(L+N-1) T} \exp (-j 2 \pi f Y(t)) \mathrm{d} t\right|
\end{gathered}
$$

For more complex forms of MTF, numerical analysis can be used to calculate.

\subsection{Image Compression}


As the new generation of still image compression standard, JPEG2000 with its excellent compression features and higher resistance have been widely used in the fields of astronautics and aerospace [11].

\subsection{Image Restoration}

In the process of remote sensing image generation, storage and transmission, often because of hardware equipment, weather conditions, light intensity, motion blur, noise. camera mis-focus and a number of factors such as image quality degradation. Image restoration is performed by reversing the process that blurred the image and such is performed by imaging a point source and use the point source image, which is called the Point Spread Function (PSF) to restore the image information lost to the blurring process. In order to recover these corrupt images, a number of mature image restoration methods, including inverse filtering, Wiener filtering [12], constrained least squares filtering and Lucy-Richardson(LR) filtering [13,14].

This paper proposes an adaptive modulation transfer function compensation (MTFC) restoration algorithm of remote sensing images based on spatial domain [15]. The method is based on the modulation transfer function values laboratory-measured, which considers the impact of optical system, detector and electronic components, and construct finite impulse response to calculate deconvolution nuclear. According to the signal to noise ratio (SNR) of different gray values given by the sensor parameters (including integral series, gain, etc), we handle image deconvolution, which combining scene content for adaptive noise suppression processing. The method can improve both the image clarity and the SNR to increase the image quality.

\section{Remote sensor optimization design}

Considering the influence of each link, such as the scene, atmosphere, remote sensor, satellite platform and image compression on the imaging quality, at the same time, the restoration process is added, the parameters which affect the imaging quality are analyzed synthetically, and the design parameters is iterated for several times. Finally, the optimal design of remote sensing system is achieved. Figure 2 is Optimization design flow chart.

Specific steps are as follows.

(1) According to the needs of users, the five main factors affecting the capability of remote sensor, such as GSD, Swath width, MTF, SNR, Aliasing, are used as the input parameters of the simulation system.

(2) According to the imaging conditions and system parameters of remote sensing system, the scene models with ground objects are used to simulate the radiation images.

(3) On the basis of the mathematical modeling of each segment of the whole link, the remote sensing imaging simulation is realized, and the system designed parameters are determined.

(4) The simulation image is restored.

(5) The restored image is evaluated to determine whether the image quality meets the requirements, and if the requirements are not met, the results are fed back to the system to design new parameters.

(6) The system parameters are evaluated to determine whether they meet the requirements of engineering development, and if the requirements are not met, the results are fed back to the system to design new parameters.

(7) When the image quality and system performance are all meet the requirements, the design parameters is obtained.

The image quality evaluation parameters in step (5) are measurable, including image mean, standard deviation, contrast, definition, detail signal energy, SNR, MTF, etc., which belongs to the objective evaluation criteria. There are some other evaluation parameters that need to be developed according to the requirements of the optical spectrum and application field.

The parameters in step (6) belongs to the remote sensor, including focal length, F-number, aperture, spectral bands, transmittivity, field of view, quantization bits, circuit noise, etc. 


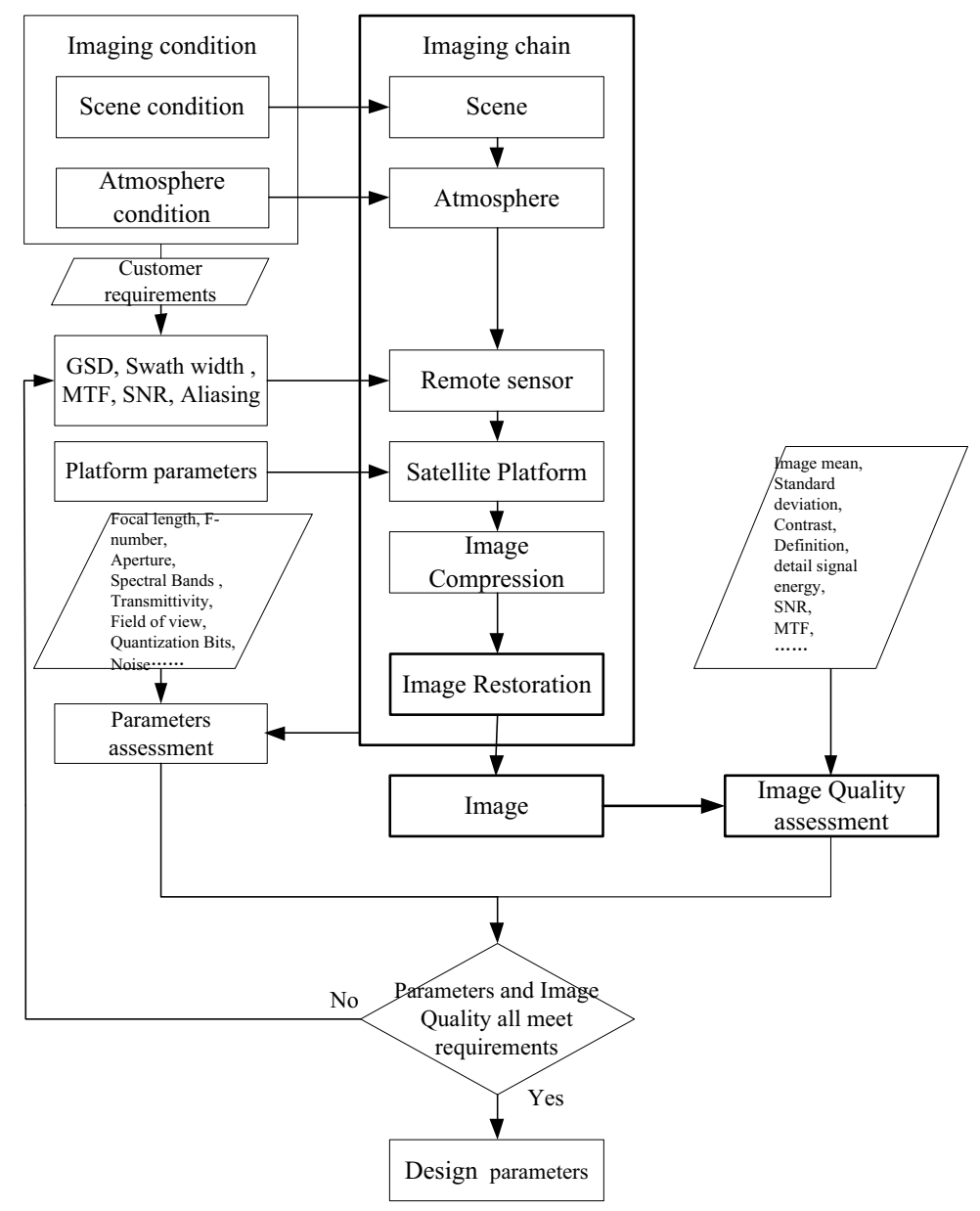

Figure 2. Optimization design flow chart

\section{Experimental analysis}

\subsection{Traditional Design}

Suppose the user needs for earth observation: the ability is $0.7 \mathrm{~m}$ resolution and $12 \mathrm{~km}$ swath width at $645 \mathrm{~km}$ height.

According to the traditional design process, in order to shorten the focal length, the pixel size of TDICCD is chosen to be $10 \mu \mathrm{m}$, and then the focal length of the system is designed to be $9.2 \mathrm{~m}$. According to the requirements of MTF at the Nyquist frequency and SNR, the camera is determined as $\mathrm{MTF}>0.2, S N R_{\max }>46 \mathrm{~dB}, S N R_{\min }>20 \mathrm{~dB}$, the obstruction ratio of optical system is $2.25 \%$, wavefront error is $0.12 \lambda(\mathrm{rms})$. In order to meet the requirements mentioned above, the aperture of optical system is $1.2 \mathrm{~m}, \mathrm{~F}$-number is $7.6, \lambda F / p$ is 0.5 , resulting in large aperture, image aliasing serious, developing difficulty, long cycle and high cost.

\subsection{Optimization Design}

User requirements are the same as traditional design. 
According to the optimization ideas, we can know the aperture is the key design parameter, which directly affect the cost, size and weight of the camera. Considering the five main factors, such as GSD, Swath width, MTF, SNR and Aliasing, the aperture is determined by optimization design.

The TDICCD is the same as traditional design, focal length is $9.2 \mathrm{~m}$. The initial aperture is determined by the requirements of $S N R_{\max }>46 \mathrm{~dB}, S N R_{\min }>20 \mathrm{~dB}$. The MTF value of each segment is assigned by the requirements of $\mathrm{MTF}=0.20$. The system uses $10 \mathrm{bit}$ quantization.

On the basis of the mathematical modeling, the remote sensing imaging simulation is realized.With the solar elevation of 20 degrees, and the ground reflectivity of 0.1 , the radiance of $6.23 \mathrm{~W} /\left(\mathrm{m}^{2} \cdot \mathrm{sr}\right)$ is used as the minimum input. With the solar elevation of 70 degrees, and the ground reflectivity of 0.6 , the radiance of $71.9 \mathrm{~W} /\left(\mathrm{m}^{2} \cdot \mathrm{sr}\right)$ is used as the maximum input. The SNR of different apertures and TDI stages is shown as Table 1 and Table 2.

Table 1. the $S N R_{\min }(\mathrm{dB})$ of different apertures and TDI stages

\begin{tabular}{|c|c|c|c|c|c|c|}
\hline \multirow{2}{*}{$\begin{array}{c}\text { Aper } \\
\text { ture } \\
(\mathbf{m})\end{array}$} & \multicolumn{6}{|c|}{ TDI stages } \\
\cline { 2 - 7 } & 12 & 24 & 36 & 48 & 72 & 96 \\
\hline 0.72 & 27 & 30 & 32 & 33 & 35 & 36 \\
& .5 & .5 & .3 & .5 & .3 & .5 \\
\hline 0.65 & 25 & 28 & 30 & 32 & 33 & 34 \\
& .8 & .8 & .6 & .0 & .6 & .9 \\
\hline 0.61 & 24 & 27 & 29 & 30 & 32 & 34 \\
\hline
\end{tabular}

Table 2. the $S N R_{\max }(\mathrm{dB})$ of different apertures and TDI series

\begin{tabular}{|c|c|c|c|c|c|c|}
\hline \multirow{2}{*}{$\begin{array}{c}\text { Aper } \\
\text { ture } \\
(\mathbf{m})\end{array}$} & \multicolumn{6}{|c|}{ TDI stages } \\
\cline { 2 - 7 } & 12 & 24 & 36 & 48 & 72 & 96 \\
\hline 0.72 & 40 & 43 & 44 & 46 & 47 & 49 \\
& .0 & .0 & .7 & .0 & .8 & .0 \\
\hline 0.65 & 38 & 41 & 43 & 44 & 46 & 47 \\
& .4 & .4 & .2 & .4 & .2 & .4 \\
\hline 0.61 & 37 & 40 & 42 & 43 & 45 & 46 \\
& .2 & .2 & .0 & .2 & .0 & .2 \\
\hline
\end{tabular}

In order to reduce the difficulty of engineering development, Static MTF was reduced to 0.1 , after the restoration process, MTF increased to 0.2 . It is also necessary to consider whether the aliasing of the image is within the allowable range. This process requires compromise and optimization. The simulation results show that the aliasing parameters $\lambda F / P$ are between 0.8 and 1.2.

With the image mean, standard deviation and the detail signal energy as evaluation parameters, the simulation image quality of different F-Number is shown in Table 3 . The mean represents the brightness of the image. The standard deviation represents the richness of the gray level of the image, and the detail signal energy represents the richness of the detail signal in the region.

Table 3. The image quality evaluation of different F-Number

\begin{tabular}{|c|c|c|c|}
\hline $\begin{array}{c}\text { F- } \\
\text { Number }\end{array}$ & $\begin{array}{c}\text { image } \\
\text { mean }\end{array}$ & $\begin{array}{c}\text { standard } \\
\text { deviation }\end{array}$ & $\begin{array}{c}\text { detail } \\
\text { signal } \\
\text { energy }\end{array}$ \\
\hline 10 & 63.3 & 22.3 & 124.8 \\
\hline 12 & 59.5 & 19.8 & 104.4 \\
\hline 14 & 55.1 & 17.7 & 81.1 \\
\hline 15 & 54.0 & 16.1 & 76.8 \\
\hline
\end{tabular}




\begin{tabular}{|l|l|l|l|}
\hline 20 & 38.9 & 10.0 & 34.8 \\
\hline
\end{tabular}

As can be seen from Table 3, the larger the F-number, the smaller the image mean, standard deviation and the signal energy, and the worse the image quality.

After the image quality and system performance evaluation, considering the requirements of miniaturization and stabilization, the parameters of remote sensor is designed as: aperture is $0.65 \mathrm{~m}, \mathrm{~F}$ number is $14, \lambda F / p$ is 0.9 . The simulation results are shown in Figure 3. Figure 3 a) is the result of traditional design simulation. TDI stages is 12 , aperture is $1.2 \mathrm{~m}$, and F-number is 7.6 , Static MTF is 0.2 . Figure $3 \mathrm{~b}$ ) is the result of optimization Design simulation. TDI stages is 72 , aperture is $0.65 \mathrm{~m}$, and F-number is 14 , static MTF is $0.1, \mathrm{MTF}$ after restored is 0.2 .

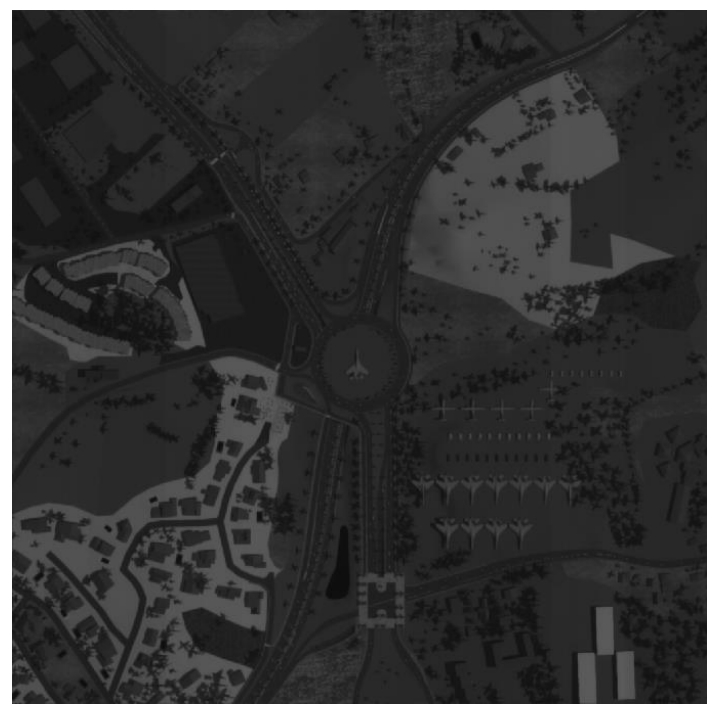

a) Traditional Design

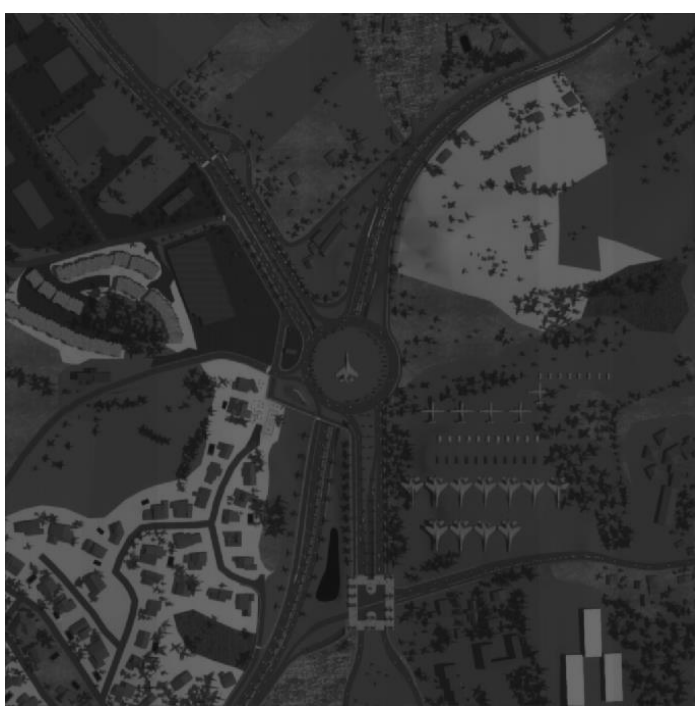

b) Optimization Design

Figure 3. Simulation image

The final design parameters are shown in Table 4.

Table 4. design parameters

\begin{tabular}{|c|c|}
\hline Orbit Height $(\mathrm{km})$ & 645 \\
\hline GRD $(\mathrm{m})$ & 0.7 \\
\hline Swath $(\mathrm{km})$ & 12 \\
\hline Pixel Size $(\mu \mathrm{m})$ & 10 \\
\hline Focal length $(\mathrm{m})$ & 9.2 \\
\hline Aperture $(\mathrm{m})$ & 0.65 \\
\hline F-Number & 14 \\
\hline$\lambda F / p$ & 0.9 \\
\hline MTF Static MTF & $\begin{array}{c}\text { Before compensation } 0.10 \\
\text { After compensation } 0.20\end{array}$ \\
\hline$S N R_{\max }(\mathrm{dB})$ & $>46($ TDI stages is 72$)$ \\
\hline$S N R_{\min }(\mathrm{dB})$ & $>20$ \\
\hline
\end{tabular}

According to the requirements of the swath with, the optical system field of view is determined. The design scheme of optical system is proposed based on the aperture, focal length, pixel Size, etc. The next step is the detailed design of remote sensor. 


\section{CONCLUSION}

In this paper, based on the end to end simulation, the optimization design method for remote sensor is studied. The parameters which affect the imaging quality are analyzed synthetically, and the design parameters are iterated by the evaluation of image quality and system performance. Finally, the optimal design of remote sensing system is achieved. The test validates the feasibility and predominance of the optimization design method. Using this method, the remote sensor can meet the requirements of the engineering development and image quality. The aperture of the remote sensor is reduced by about $1 / 2$, and the development difficulty is greatly reduced.

\section{References}

1. Edson Robert, Frittman Jaime. Illustrating the Concept of Operations (CONOPs) Continuum and Its Relationship to the Acquisition Lifecycle[C]. 7th Annual Acquisition Research Symposium to be held May 12-13, 2010 in Monterey, California. U.S.Government or Federal Rights License.

2. Amundsen R M; Feldhaus W S; Little A D; Mitchum, M. V. Integration of design, structural, thermal and optical analysis and user's guide for structural-to-optical translator (PATCOD)[C]. Mar 1, 1995 Langley Research Center. NAS 1.15110153; NASA-TM-110153 95N24559.

3. John $M$ Irvine. National imagery interpretability rating scales (NIIRS): overview and methodology[C]. Proceedings of SPIE, 1997, Vol.3128, 93-103.

4. Taejung Kim, Hyunsuk Kim, HeeSeob Kim. Image-based estimation and validation of NIIRS for high-resolution satellite images[C]. The International Archives of the Photogrammetry, Remote Sensing and Spatial Information Sciences(ISPRS), Vol. XXXV II, Part B1. Beijing 2008.

5. Leachtenauer J C, Malila W, Irvine J, Colburn L. General Image-Quailty Equation: GIQE[J]. Applied Optics, 1997, 36(32): 8322-8328.

6. Leachtenauer J C. Image Quality Equation and NIIRS[C]. Encyclopedia of Optical Engineering, 2003.

7. Ryan R, Baldridge B, Schowengerdt R A, Choi T, Helider D L, Blonski S. IKONOS spatial resolution and image interpretability characterization[J]. Remote Sensing of Environment, 2003, 88(01):37-52.

8. Philippe Kubik, Veronique, Paseal, Francoise de Lussy. Pleiades-HR image quality design[C], ACSM-ASPRS 2002 annual conference proceedings.

9. Krapels K, Driggers R G, VolImerhausen R H, et al. Atmospheric turbulence modulation transfer function for infrared target acquisition modeling[J]. Optical Engineering(S0091-3286), 2001,40: 1906-1913.

10. Kobi Buskila, Shay Towito, Elad Shmuel, et al. Atmospheric modulation transfer function in the infrared[J]. Applied Optics (S0003-6935), 2004, 43(2): 471-482.

11. ISO/IEC. JPEG2000 Image Coding System: Part1-Core Coding System. ISO/IEC FCD15444-1. 2000:118-127.

12. R. C. Gonzalez \& R. E. Woods, Digital Image Processing , Addison-Wesley Publishing Company, Inc., 1992.

13. Biggs, D.S.C. Acceleration of Iterative Image Restoration Algorithms. Applied Optics. Vol. 36. Number 8, 1997, pp. 1766-1775.

14. Hanisch, R.J., R.L. White, and R.L. Gilliland. Deconvolution of Hubble Space Telescope Images and Spectra. Deconvolution of Images and Spectra (P.A. Jansson, ed.). Boston, MA: Academic Press, 1997, pp. 310-356.

15. ZHOU Nan, QI Wenwen, CAO Shixiang, HE Hongyan, XING Kun, YUE Chunyu, Adaptive MTFC Restoration Algorithm of Remote Sensing Images Based on Spatial Domain , SPACECRAFT RECOVERY \& REMOTE SENSING., 2015, 36(4):54-62. 